\title{
Explicating the Challenges of Providing Novel Media Experiences Driven by User Personal Data
}

\author{
Neelima Sailaja, Andy Crabtree, Derek McAuley \\ University of Nottingham \\ Nottingham, UK. \\ first.last@nottingham.ac.uk
}

\author{
Phil Stenton \\ BBC R\&D \\ Salford, UK. \\ phil.stenton@bbc.co.uk
}

\begin{abstract}
The turn towards personal data to drive novel media experiences has resulted in a shift in the priorities and challenges associated with media creation and dissemination. This paper takes up the challenge of explicating this novel and dynamic scenario through an interview study of employees delivering diverse personal data driven media services within a large U.K. based media organisation. The results identify a need for better interactions in the user-data-service ecosystem where trust and value are prioritised and balanced. Being legally compliant and going beyond just the mandatory to further ensure social accountability and ethical responsibility as an organisation are unpacked as methods to achieve this balance in data centric interactions. The work also presents how technology is seen and used as a solution for overcoming challenges and realising priorities to provide value while preserving trust within the personal data ecosystem.
\end{abstract}

\section{Author Keywords}

Media; personal data; data; interviews.

\section{ACM Classification Keywords}

Human-centered computing Field studies

\section{INTRODUCTION}

With media becoming increasingly online and ubiquitous, it has shifted from traditional scenarios of audiences consuming from service provider dictated schedules to the freedom of consuming customised and context-sensitive content available at the user's demand. This transformation has led to a turn towards personal data to help media service providers[39] meet audience expectations.

Research has identified a number of challenges introduced by the use of personal data which include loss of user

Paste the appropriate copyright/license statement here. ACM now supports three different publication options:

- ACM copyright: ACM holds the copyright on the work. This is the historical approach.

- License: The author(s) retain copyright, but ACM receives an exclusive publication license.

- Open Access: The author(s) wish to pay for the work to be open access. The additional fee must be paid to ACM.

This text field is large enough to hold the appropriate release statement assuming it is single-spaced in Times New Roman 8-point font. Please do not change or modify the size of this text box.

Each submission will be assigned a DOI string to be included here. trust[20], privacy compromises[44] and asymmetry of power over data[25]. While there is a growing call within academia to explore the socio-technical underpinnings of data[7], the consequences of these challenges within the domain of new media, are yet to be explored.

Recent research heralds a number of diverse opportunities pioneered by the use of diverse types of user data[5,13,34]. While such research contributes primarily to the design and evaluation of the user experience of new media experiences, the challenges and shifting priorities presented by the use of data to power these innovations are not often accounted for. To ensure sustainable growth of data driven media technologies, there is a need for understanding the drivers and challenges shaping their growth. Here, there is an explicit call to unpack and understand these priorities to help explore solutions that maximise the potential of personal data while minimising the challenges introduced by it.

This paper takes up this challenge by studying the presentday practices within a large U.K. based media organisation which has very recently shifted to technological frameworks powered by personal data. As part of this initiative, we interviewed employees working in varying capacities, serving and managing a diverse range of data driven media services.

The results of this study, while deeply rooted in varying perspectives and contexts, reveal the unified need for improved interactions in the user-data-service ecosystem where user trust and value are to be consistently balanced to ensure sustainable innovation in data driven new media experiences. While maintaining this balance manifests in diverse manners for different stakeholders, three priorities were identified as potential pathways to ensure the same. The results unpack being legally compliant and going beyond just the mandatory to further ensure social accountability and ethical responsibility as an organisation as the means to achieve and preserve this balance. Here, technology is often seen and utilised as a solution for overcoming challenges and realising priorities successfully to ensure sustainability within the personal data ecosystem.

Thus, the contributions of this paper include :

- Explicating the need for better interactions in the user-data-service ecosystem in data driven media 
experiences, where trust and value ( to both the user and the service provider ) are balanced.

- Unpacking how various legal, social and ethical considerations play out as key contributors that could help achieve and maintain this balance.

- Presenting how technology is used as a mechanism to embody solutions that preserve this balance.

\section{BACKGROUND}

\section{Personal data and new media}

The utilisation of personal data to drive new media experiences translates itself into a shift from traditional forms of broadcasting to a mass audience towards the practise of narrowcasting where individual users with unique user profiles are provided with tailored and interactive user-specific experiences.[6,10,19,22].

Interactivity in media has traditionally been a muchdeliberated concept. Steur's definition of interactivity in a media experience was one where 'users can participate in modifying the form and content of the mediated environment in real time' [48]. Markus's version of the same stated that an interactive medium is a vehicle that enables and constrains multidirectional communication flows among the members of a social unit'[29]. Rada added the capabilities of computer-mediated information sharing systems including multi-media and hypertexts[35]. Today, personal data has emerged as an enabler that helps realise all of these definitions of interactivity to provide users with multifaceted interactive experiences that removes 'old constraints' and offers users 'new liberties of action'[11], through interactivity that respects every individual user's requirements.

Examples of such experiences are dissimilar in nature with respect to the data collected, sources of collection, use of data and the very essence of the experience itself. There are movies that adapt themselves continuously to user reaction learned through real time physiological data[34]. Formats where news programmes are personalised on user televisions to provide more relatable and relevant content, are being explored[5]. Personalisation of broadcast[12], a necessary methodology and tool that supports varied forms of tailored experiences are also currently gaining much momentum in media research. Other forms of experience enhancement supported by personal data include tailoring of recommendations [2], personalised electronic programme guides[24], recommendations made based on user emotions[13] etc.

\section{Current Challenges}

While the use of personal data leads to considerable appreciation in technological growth in media, the shift also entails caveats that dictate careful management of the many challenges this shift introduces into the system.

While personal data affords "new horizons for personalised, interactive and immersive entertainment as well as marketing...[...]... At the same time, some such proposals may be at variance with human values many of us hold dear, including privacy, trust, and control."[18]

A World Economic Forum report on personal data[20] details the current crisis in trust associated with the service providers' use of personal data. "The widespread loss of trust is unmistakable: security breaches, identity theft and fraud; concern from individuals and organizations about the accuracy and use of personal data; confusion from companies about what they can and cannot do; and increasing attention and sanctions from regulators.'"[20]

In 2006, Netflix was forced to cancel the sequel to their \$1 million personal data driven recommendation algorithm improvement contest[47] following a warning from privacy advocates that notified the new dataset to be easily deanonymised. This was also coupled with a suit filed by a privately homosexual mother who was "alleging that Netflix violated fair-trade laws and a federal privacy law protecting video rental records, when it launched its popular contest in September 2006"[46], further affecting user trust in media organisations adversely. The current political allegations of 'fake news'[51] also renders itself into this scenario where users are questioning media organisations and their trustworthiness, leading them to depend on alternate sources of news media like Facebook, Twitter and YouTube that delivers more user generated content, potentially undermining the situation further, as these channels are not subject to media regulation.

Along with the challenges of loss of privacy, trust and legal implications is the lack of control given to the user. Lanier[25] had mentioned an asymmetry of control in the digital economy where the users, who are both the producers and consumers of the data commodity, exercise very little control over it. While research has highlighted the importance of allowing data legibility, negotiability and agency through control[32] to the users, there still remains an explicit call for the technological embodiment of these principles. Users are continued to be presented with incomprehensible privacy statements[31,37], followed by acceptance statements where the users are constrained to binary choices[45] dictated by service providers often leading to low levels of understanding and very little space to exercise control[43].

Therefore, in order to ensure continued innovation in media research supported by user personal data, there requires a better understanding of the current landscape of the personal data ecosystem, it's priorities, opportunities and challenges. This work intends to explore these domains by identifying the current challenges and potential methods and pathways that help overcome these challenges to ensure sustained innovation in data driven media experiences.

\section{THE STUDY}

The study method consisted of interviews and was performed in collaboration with a large publicly funded media organisation within the U.K.. After years of 
resistance, the organisation is currently in the process of shifting to mandatory user sign-ins and collection of personal data. They serve a diverse variety of media experiences catering to audiences of varying demographics. This includes News, Sports, Children's entertainment, Knowledge and Learning, Radio, Video on Demand, 360 experiences, Virtual Reality experiences, Live Broadcast etc.

\section{Participant Recruitment}

The recruitment began with the help of a gatekeeper who enabled access to certain key teams. The majority of the participants were contacted individually through recruitment emails that outlined the aims of the research and their involvement in it. The rest were recruited by snowballing references through participants who identified potential candidates who worked within the same team, but in a role starkly different from theirs.

\section{Interview Method}

Since the participants hailed from teams that served vastly different kinds of experiences and hence had different priorities, in order to successfully achieve the goal of the study, the interviews had to accommodate the diversity in the views of the participants. Simultaneously, the interviews also had to accommodate dialogue around the issues that the study aimed to learn about.

These priorities resulted in an interview design which was unstructured, consisting of a set of topics that helped guide the conversations if they ever stalled[38]. The three areas of interest that were used as seeds to drive the conversations were:

- the current collection and use of personal data,

- the benefits and risks of using personal data, and

- the future of personal data in new media.

The conversations flowed where the respondents directed them making the method "informant"[27] in nature. In instances where topics that were aimed to be discussed were ignored, the researchers ensured that they seeded them into the conversations through questions, follow up questions, comments or queries. Given the highly complex nature of the topics discussed wherein the attitudes towards personal data are wide and varying with the reasoning for the same being highly contextual, we believe the exploratory approach helped accommodate the complexity and multifaceted nature of the data in its full richness.

With the view of using the results of the study in design endeavours that would enable future data driven media experiences, the conversations were often encouraged to be deeply rooted in current technologies that the participants were contributing to. This helped demonstrate the current practises and also worked as probes[17] to visualise future scenarios that the participants envisaged would help overcome the current challenges they face.

\section{Data Gathering}

A total of 20 interviews were conducted with stakeholders who work in diverse teams, which included Sports, News, Children's content, Education, Video-on-Demand platforms, Radio, Research and Development, Audience Platform, Marketing and Audiences and Data Management. These participants serve varying roles within these teams which includes engineers, technical architects, digital planners, analysts, UX practitioners, designers, producers etc. These participants also work in diverse capacities and hierarchical levels within the organisation which includes, directors and executives (4), department leads and heads (5), senior professionals (7) and relatively newer recruits (4). The group included 6 females and 14 males with ages ranging from $20 \mathrm{~s}$ to $50 \mathrm{~s}$. This mix of teams and experience ensured representation of the varying priorities raised at different levels of work organisation while serving a wide range of media experiences. The common connection that served in the selection of participation was the individual's involvement with data driven technologies and personal data.

The interviews were either in person, by telephone or Skype, and lasted thirty to forty five minutes. All interviews were audio recorded and the recordings transcribed for data analysis purposes. The data collection and transcription was done simultaneously where the collection was discontinued once the data reached saturation, as the responses and discussions became repetitive.

\section{Data Analysis}

Reflecting Sacks' analytic commitment[40,41] we believe "that the warrant for any given assertion should be visible in the data"[36]. Hence, the analysis of transcribed data was done in a qualitative data driven manner. Owing to the open ended and unstructured nature of the conversations and the varying contexts addressed by the participants, the topics of discussion and the data formed a spectrum of perspectives. Therefore, we adopted endogenous topic analysis[36] through close reading of the transcripts to identify discrete topics that had manifested in the participants talk that demonstrate how they reason about personal data and its challenges.

In the first round of analysis, all topics discussed by the participants were marked out. In the second round, common topics of discussion were grouped while those that did not overlap were treated with equal weight independently. The focus here was to attend to the talk's topics, which is different from traditional forms of open coding where the text is analysed and any interesting phenomena in the data is coded and marked up[27]. By adopting endogenous topic analysis, it is not the questions that are relevant any more but the responses and how they are opened up. This helps eliminate one of the weaknesses of traditional grounded theory, the researchers' pre-conceived notions of what is "interesting"[27] and what is not. Instead, the onus is on the data itself as it churns out the topics it has embodied within 
it. Theoretically, this is in line with the documentary method of interpretation used in sociology, which in Mannheim's words is a "search for an identical homologous pattern of meaning underlying a variety of totally different realizations of that meaning."[28] Here, an actual appearance is considered "as the "document of", "as pointing to", as "standing on behalf of", a presupposed underlying pattern [14]."

This ethnomethodologically inspired method[4] also helped accommodate the heterogeneity in the topics discussed, due to the diversity in participants' roles associated with personal data. It ensured that the very unique practical reasonings presented in the data were not discarded because of lack of overlap or absence of patterns (cf. traditional thematic analysis[8]). In contrast, here the contextual distinctions help form, support and add to the richness of the emerging topics. This is a deviation from the "development of concepts"[27] stage in grounded theory analysis where a metric of evaluation of the outcome is "the number of instances in the data that support the specific concept"[27]. Instead, the analysis here is sensitive to the situational variations embodied within the data, which might otherwise be considered outliers or distractions, due to lack of overlap.

Trying to reduce and transform this data moves away from the goal of efficiently mapping this emerging scenario of personal data use in media, which demands the need for exploration that is rooted in the practicalities of the real world.

\section{RESULTS}

In the following section we present the results of the data analysis catalogued under five major topics that emerged from the interviews.

\section{Business Priorities}

The results show that the use of personal data helps achieve and regulate a range of organisational business priorities.

\section{Fulfilling the business mission}

Ensuring delivery of valuable service to every single user is one of the organisation's premier missions. Personal data is expected to become a key tool in this process.

"It has to do especially with the [ $\left.{ }^{* *}\right]$ 's mission of delivering value to everyone. So, by having an idea of the location and age and gender, we can make sure that we are delivering something for everyone.[P12]"

Use of personal data is expected to shift the relationship between the service provider and the user from the antiquated one to many broadcast model to one to one relationships that cater to each users' particular needs and interests.

"Optimally the world is changing where rather than one to many, its one to one relationships that organisations create with their audiences and customers. And so, we have to move into that world. [P14]"
Personal data provides opportunities to understand consumption trends across various demographic segments, based on age, location, etc. This helps as an evaluative tool in learning how different content is consumed across the spectrum of audiences, identifying gaps in consumption and making amends to future content creation to ensure consumption across all cohorts.

"And so we also use this case for tracking consumption, so that we can say how well we are doing on a geographic basis, and also, to an extent, if we cross reference against other available data sources, so demographic, using the geo-demographic dataset. So there's some element of use within the product, the digital products themselves, but also a great deal of value from a reporting point of view.[P2]"

With the introduction of social media, measuring reach of services is not contained within service provider platforms. Media is liked, shared and discussed by users through multiple social media platforms. Understanding who a user is, is expected to help reach out to social media profiles, providing possibilities for exploring the reach of the organisation's services on these profiles and platforms.

\section{Provide symmetrical exchange of value}

Providing users with a good exchange of value is vital to the success of any business. Provision for user accounts and profiles are expected to give users opportunities to express themselves better online and to be more active participants in the data economy. This enhanced contribution from the users could then be reflected to them in the experiences that they receive in return. These reflections could go beyond traditional media services to educational initiatives that help with personal growth, self-reflection programmes that help the user make sense of his/ her personal data with regards to contexts frequented by the user, interactive experiences that show a comparison of user data against the rest of the audiences etc.

"So, we are going around public service remit for data so where Amazon and commercial organisations it is very much about how they can market it back to you, so our question was what was a public service organisation, that's not making money do? So, we are looking at what's the most meaningful feedback? If you give us your data, how can we give you something back? That is enhancing for you, as opposed to just for us.[P8]"

\section{Help with product development}

Product development in media is based on input from many different sources within the organisation with the user having very little participation in the process. With the use of personal data, users can play a more central role in this process with the service providers being able to understand user needs down to the granularity of the specific devices used.

"So, I guess it informs the development of the product. So, it basically helps to inform staff, so for example, this is the kind of stuff that can help tell us, you know, TV platforms for example, are becoming more and more, are becoming a bigger and bigger part of [Video on Demand] usage. That's then really important for us to say, ok, should we then be putting in more refills on TV, should we be trialling more stuff on TV, that kind of thing.[P4]" 


\section{Staying relevant}

With the high level of entropy in the number of products and services that are available to users, it is an existential need for every business to stay relevant in the users' everyday lives in order to ensure their sustained success and growth. Personal data, is currently considered to be a tool that would help understand the users' changing wants and needs and hence help the business and its products stay relevant to the user in the current day.

"I also think that there's a risk though in a sense to avoiding collecting personal data ............... I think what l'm saying is that consumers demand personalised experiences and that requires personal data. And if you cannot supply them what they expect, then they will consider you to be irrelevant.[P3]"

\section{Importance of Trust}

Trust was stressed as one of the key values of the organisation and as an international brand, it represents a public service entity that has always worked in ways that upheld user trust as a priority. This trust, that has been built over the years, is part of the organisation's legacy and is very important to the organisation. As a business, they recognise the possible erosion of user trust the mismanagement of user personal data could lead to and hence are very careful that their experiences reflect this value at all levels and dimensions.

"There's another risk which is.... and this is back to our relationship with our audience. So, we, the $\left.{ }^{* * *}\right]$ is a very well trusted brand and you know to maintain that trust we need to ensure that we are not doing anything weird with the data that people provide to us.[P2]"

\section{Legalities of using personal data}

The General Data Protection Regulation was referred to as a guide for designing future media experiences. It was acknowledged that future regulation supports provision of higher levels of data legibility to the user which is aimed to empower the data subjects with an understanding of data practises around services offered by the organisation. This puts more pressure on service providers to deliver valuable services where the need, use, consent and control of personal data is easily justifiable not just to the user but legally as well.

"I think what the GDPR is bringing is a bigger literacy about us, of users giving away our data to get better benefit, but we need to be conscious about to whom we give that data and what is the actual value we get from that. Because if we are much more literate, people will have to treat our data with care, with much more care. And if we end up in a situation where this information would be lost, that would be a bad thing for the organisation and we might just lose completely, the trust.[P5]"

\section{Personalisation}

Enhancement of experiences and user journeys through personalisation was identified as one of the key uses of personal data in new media. The creative contribution of personalisation in crafting current and future experiences was often acknowledged, the legal implications of the same was also recognized.

\section{Personalising the media experience}

Personalisation is used to enhance new media experiences in various ways including both personalisation of recommendations and notifications, as well as the content served, e.g., video, audio, news, sports etc.

"It's not about recommendations like, because we know about you, you might like to buy this book. It's more like we know that you live in wherever it is you say that you live in off Facebook and we know that the main football team there is and that kind of thing. So the football team in the story, it's the team that's closest to you....we think you like that.[P3]"

Personalisation is also extended to scenarios where the user collaboratively engages with the experience to drive it in a unique and personalised fashion. An example of this is a "cook along" kitchen experience where data from smart utensils and/ or user input are used to continuously tailor the content to match user expertise and pace.

\section{Personalisation for enhancing user journeys}

Personalisation is expected to help in the process of exposing the audiences to the breadth of the content available to them.

"So, you know we have many many hours of TV and radio every single day. Both international and regional radio stations and TV networks. And we create about one and a half thousand new webpages every single day. Most of all that content..... it would be impossible for any individual to be across it all......]... Its just packages of information or content, that may well be of interest to an individual but they are not going to know that its even available... I mean in the digital era just because something goes up in a regional radio station, it might be about a subject that somebody who doesn't live in that region is interested in. [P14]"

Personalisation also helps go beyond just the serving of content of interest. It could also be applied to curate and enable entire user journeys to suit the users' wants and needs.

Personalisation introduces the possibility of new "genres of experiences" which otherwise would not have been possible. Each user, through their own, unique set of personal data would be able to create and experience exclusive and novel genres of experience.

"What it does is that every day it gives you sort of an on this day in the past view of your social media accounts in the past and what you posted.............I think what I guess what I'm trying to get at there is that's a whole experience that's based only on my personal data and it's based on my personal data not yours. Or rather yours is based on your personal data and mine is based on mine. And it's a kind of genre of experience that I think is very powerful.[P3]"

Personalisation and the Law

In the U.K. it is expected that not all individuals are exposed to the same degree and type of personalisation. Service providers are sensitive to this gradation in personalisation services and are considering design alternatives that accommodate such legal constraints.

"In the UK there is a, we have this idea of not every single user will get the personalisation on the same level as others. In line with the future regulation GDPR, we would not be able to serve personalised 
recommendations, personalised experiences, without the consent of a parent or a guardian, for anyone under 13. So, currently, for under 13 years old have their personalisation disabled by default which means that when they register and they are signed in, they are not receiving any personalised experiences as we are not able to use any of their data for personalisation.[P5]"

\section{Crafting future content}

Personalisation is considered as a "research tool for the creative process[P5]". It could help rethink the crafting of future content creation to help enhance experiences.

"Its more giving the content commissioners the capability, tools and information to enhance the decision making process. To be more in line with what people actually need. And then, as a result of that, we are much more likely to target better content. And show a wider content which might be in line with peoples' kind of expectation.[P5]"

\section{Forming Cohorts}

Forming user cohorts based on demographic data or user behaviour was often discussed as a method of leveraging personal data that afforded a range of possibilities, like abiding by legislation, delivering relevant content and evaluating the reach and response to disseminated content.

\section{To abide by legislation}

The formation and use of user cohorts based on age was adopted to ensure alignment with legislative constraints regarding the different types of personal data that was collected from signed up users. Cohorts also help manage users' age transitions, which is accompanied by changes in the jurisdiction with respect to their personal data use.

"For children under 13 we do not collect full post code, we collect town, the town where they live. And, we also, for practical purposes today, if you are under 18 we collect your year of birth, sorry your date of birth so that we can work out when you transition from being under 13 to over 13 and when you transition from being under 18 to over 18, so that we can track those transitions. [P2]"

Cohorts also ensure that every user has, by default, available to him/ her the legally allowed grade and form of services for their age.

\section{To deliver relevant content}

Cohorts help target content that is relevant to users. Targeting is enabled across diverse types of content, including television shows, online programmes, radio shows and even marketing emails that are served to audiences. Forming cohorts helps with the personalisation of these varied types of content to make more targeted decisions about the material to be served to a user based on his/ her membership in various cohorts.

"So, personalisation comes in many types and forms. So, it's creating um, improved websites, it improves programming, it's giving recommendations to people what they might want to watch, depending on which cohort they sit in and even sending newsletters out to people. So, if I, for example, if I'm middle aged and if I really like nature programming and I like Radio 4, but I don't like Sports, why would you be sending me emails about sports or why would you be sending me emails about Radio 1 extra when you know that I'm a Radio 4 listener.[P10]"

\section{To measure response and reach}

Cohorts also perform the function of evaluation of disseminated content. It is used "to measure our conversion rate and measure how we succeeded with the making of better experiences.[P5]" It also helps to highlight gaps in content consumption, especially helping uncover flaws that might be preventing delivery of service to specific sets of audiences that belong to particular demographic groups.

"In terms of developing new services, understanding which bits of those new services are reaching audiences that we are not currently reaching. Or we are just building things that are super serving our existing audiences. Helping us understand if there are gender imbalances, if we are serving people in different parts of the world in different ways. You know, we are doing particularly well with women in the South of England but we are doing very poorly with women in India.[P16]"

\section{Risks}

Concerns like reputation damage, loss of user trust and data security concerns were flagged as potential risks of using personal data. Current risk mitigation measures like anonymisation, encryption and prioritisation of transparency were also discussed in parallel.

\section{Reputation Damage}

The potential of personal data, if not used in a judicious manner leading to possible maligning of reputation was highlighted as a risk.

"The main risk is that you could really screw up as an organization............... And your reputation would be ruined as an organisation you know.[P3]"

The stakeholders recognise their role as a data controller and the importance of being legally and ethically responsible for their use of personal data, so that the legacy and reputation of the organisation is preserved.

"Ok legal risks, in the sense of, if we are thinking in terms of the audience then, in the data protection law, we are the data controller, I think that's the terminology. And I think we have certain legal obligations to look after, in our role as the data controller, that we do appropriate things with people's data. And then there's also just..... I think from our own point of view, what we want to do our own ethical things as far as the [ $\left.{ }^{* *}\right]$ 's reputation is at risk.[P17]"

Trust

While the importance of using personal data is undeniable, there is also the risk of being intrusive as a service provider during the collection and use of this data. They consider being "creepy[P2]" as a risk that would undermine the trust the audiences have on the organisation and its services.

"So, we, the $\left[{ }^{* * *}\right]$ is a very well trusted brand and you know to maintain that trust we need to ensure that we are not doing anything weird with the data that people provide to us...[...]... And we also have the informal mission of don't be creepy.[P2]"

\section{Constraining and biasing user attitudes}

An interesting comparison of a user's browsing space in the internet to that of a neighbourhood, helped highlight the risk of such personal data being used to bias and change 
user behaviour to match and benefit organisational expectations.

"If you think about back in the days, you lived in a neighbourhood, this neighbourhood has a specific group of people associated with it and so socio-economic kind of status. This information has been then aggregated and sold to advertising when you are walking in a specific neighbourhood you have a different advertising. The same thing happens right now online, however, we can be much more specific. You know much more latest precision targeting specific people.[P5]"

The fear of pushing irrelevant content to groups of users based on inaccurate decisions made due to their membership in a particular cohort was also considered a risk that constrained and biased user experiences.

\section{Serving appropriate content}

The ethical need to serve users with appropriate content is further emphasised with the involvement of users below the age of eighteen. With the use of shared devices and tailored user profiles, the risks of children being exposed to content inappropriate for them is increased and calls for attention.

"I suppose the other thing is, this is probably less.... for the [***], which is a trusted brand, meaning that people have lots of faith in what we do and all sorts of consideration behind the scenes to make sure our services are appropriate for the intended audiences, you know....more so in the case of Children's content, where we go to great lengths to make sure that our online users who are children, doesn't necessarily have access to certain aspects of what we do. And then we think about parental approval etc. When you have signed in users or signed in devices that are potentially shared, there are risks there.[P14]"

\section{Creative challenge}

Another risk outlined was the creative challenge of utilizing data to its fullest potential.

"And then there's also the risk that people aren't trained well enough to understand how to use that data for the benefit of all audiences, because it's kind of a new thinking and it's new and so there's a lot of change needed in people's minds and the ability to understand how to utilise and exploit this data for good, rather than it just adding to the noise of information that's there.[P18]"

\section{Data Security}

The importance of securing the personal data collected was highlighted continuously in discussions about risks. The potential to become a target for hackers was perceived as a high-stake risk.

"If its exposed for other purposes that wasn't designed to be collected, or if it is exposed for purposes outside of the [ $\left.{ }^{* * *}\right]$ that is bad on our part both reputationally and legally that's wrong on our part. So, we have got to put lots of security around it.[P6]"

\section{Risk Management}

Along with the discussion of the various risks posed by personal data, risk mitigation strategies undertaken were also mentioned.

Anonymisation of usernames, data minimization[49,50] and encryption were all technological measures that were discussed as part of current risk management strategies.
The emphasis on transparency was also highlighted as part of the current risk mitigation process. "So, we have worked with the information commissions office to come up with a strategy that helps our users to understand how we use our data. So, our mitigation for that trust question is that we try to get really transparent in how we use the data. [P2]"

\section{User empowerment}

Dialogue around personal data renders it as a tool that enables improved interactions through user empowerment by helping understand the user, including the user in the creative processes and exploring the potential of consent models that are user-focused.

\section{Understanding the User}

One of the most notable benefits of using personal data for a service provider was its capacity to help understand users better.

"Being able to understand who somebody is at a more granular level than what you can do without being signed into something and without capturing personal data is a huge benefit. So, being able to kind of go, this worked for this kind of audience and we can prove it through kind of statistical data. That's really massive. [P13]"

Behavioural data which helps understand actual user activity and consumption on a platform would uncover user interests and habits related to media consumption. This adds considerable value to user online identities, resulting in interactions influenced by the users themselves.

Also, understanding user intentions and delivering experiences that are aligned with every user visit is expected to be a radical novelty that would help transform the current service delivery norms.

"It will, what will we use it for, again it's about providing useful experiences to people. So, if you are just wasting time, so you just want to be entertained, then we might provide you with smaller, more upbeat, more light hearted news. But if you want to be informed about a topic, then we would offer you more deeper, more related content and deeper related content to the same content that you are interested in.[P15]"

\section{Deliver to the user}

Personal data contributes to various techniques that make content delivery more efficient and appropriate to the users. Understanding the users with the help of personal data, by combining content from differing temporal, geographic and contextual sources helps collate the most appropriate experiences for every user.

"So, if we take full post code, in our digital products, we resolve that to things like what TV region are you in, so that on [Video on Demand platform] we can show you the appropriate version of your $T V$. We also use it to determine your local radio station, we also use it to determine the weather forecast for your location. And, when we have any local news or local news alerts we can update you based on your location.[P2]"

With the increase in popularity of internet television like Netflix etc, the popularity of broadcast television has seen a drop[3]. One of the biggest challenges this shift has introduced is the need to stay relevant to younger 
audiences. The learnings gained from the use of personal data is expected to help maintain relevance over all demographic bands by ensuring delivery of relevant content to everyone.

\section{Include the user in crafting future media content}

Using personal data could help involve user priorities when making decisions regarding crafting future media experiences. By using personal data to shape media experiences, the scope of decision making essentially broadens from that of just editors and content commissioners to the users themselves.

"I'm actually quite interested in moving beyond that involving users with the algorithms, instead of us trying to guess what people are interested in. I'm more interested in them telling us. And that's what so much personal data and indicating preference and storing people's preferences and being able to go back and edit those and update them as they change.[P15]"

\section{User centred consent models}

User consent is one of many legal bases for any technology to collect, store or use personal data[15]. Consent has been translated into design by allowing the users with the choice of opting in or out of personalisation. Also, to ensure user consent happens through user empowerment the need for the user to be knowledgeable about data practises was agreed upon. The provision of alternative models of layout for the organisation's privacy policy was considered a response to this scenario.

"We publish information at three different levels. In the UX, in the user experience, as you are entering your fields of why kind of question. Then you can drop down to a short summary. That makes through to a more detailed description, still written in English rather than legalese that describes what is the.... how do we use the data, why are we collecting it and that sort of thing. And then that works through to our privacy policy which is a kind of legal document.[P2]"

\section{DISCUSSION}

\section{Organisational Concern: Striking the balance}

Research has shown the use of data to increase productivity and performance[9,26], help with management decisions[30] and contrastingly, sometimes even disempowerment[7], depending upon the nature of the service provider. The results of this study reflect that while personal data used in new media technologies is a significant contributor to creativity and innovation, there is the desire for improved interactions in the user-data-service ecosystem to ensure a healthy balance between the value produced by the use of personal data and the potential loss of user trust it could result in.

\section{Value}

The value created by personal data to both the media service provider and the user is multifarious in nature. Ranging from providing novel creative affordances to helping support business interests, it is highly dependant upon the context, the audiences catered to and the services offered.
Personalisation of varying types ( providing new genres of experiences, user specific experiences, holistic experiences, curated user journeys ) is one way of providing value through enhancement of the creative aspects available. Staying 'relevant' by keeping up with competition through the use of personal data is considered another way of producing value, here, by catering to the business interests of the organisation. The ability to form and manage cohorts to support communities and extend the reach and relevance of content was also identified as a means to value derivation. Also, the availability of user personal data further unfolds the challenge of promoting the identification of alternative methods of value producing measures presented by personal data that extend beyond just the content and the business to more user-focused experiences like customised self-reflection and feedback initiatives.

\section{Trust}

While personal data increases the potential channels for value creation, it also simultaneously introduces the risk of loss of user trust, emphasising the need to foster trusted relationships with the users during this process.

Access to personal data has led to the demand for more data and an increase in the resolution of potential data. When data becomes increasingly granular and detailed, the dangers could start outweighing the benefits as users become increasingly sensitive to mismatches and errors. The ability to cohort users further introduces the risk of bad stereotyping leading to lowered user satisfaction and trust in the organisation. Also, the need to stay relevant by keeping up with competition demands constant focus on user priorities to ensure interactions that compromise on user trust are not adopted in the process. Thus, the availability of user personal data, while affording a number of possibilities, also increases the need for ensuring preservation of user trust while putting these novel methods to practise.

\section{Need for balance}

The World Economic Forum has already detailed a crisis in trust associated with personal data use in technologies[20]. Previous research also specifically demonstrates decreased levels of user trust while using media technologies driven by personal data[42].

The results of this study further uncovers various diverse practical manifestations of the opportunities and threats introduced by the use of personal data that contribute to the loss of user trust. While it affords service providers with multifarious channels for value creation, including better support for business priorities, providing increased service affordances through personalisation, understanding the users better through cohort formation etc., it also presents, in parallel, various risks that undermine user trust in the organisation. This demands the need for user empowerment through interactions that balance both trust and value in the user-data-service ecosystem. 


\section{Response Mechanisms}

This study reveals the considerations contributing to this scenario to be legal, social and ethical in nature. While the legal priorities often arise from the impending GDPR that acts as a response to the trust crisis, the findings reveal that service providers acknowledge the need to think beyond mandatory legal obligations to cater to social and ethical concerns raised by the use of personal data, in order to preserve user trust in data driven services.

Legal

Legal requirements are a prominent sculpting force while designing and delivering personal data driven technologies as they enforce accountability into the scheme in a mandatory manner. The near future introduction of the GDPR aims to bring user trust back into the digital economy through the enforcement of legislation. Hence, aligning with legal requirements plays an important role while using personal data in media experiences.

"With the younger audiences, we are allowed to keep the data in order to be able to say that so many people do this, that or the other, but we are not allowed to keep that data attributed to one person, that says because you watched this, you can then watch that, because that becomes marketing, we are not supposed to be using that data for in that sense. So, there are all sorts of legal frameworks that we are trying to sort of... to work within.[P18]"

In the U.K., legislation dictates stark distinction between the grade of personal data collection and personalisation served to users under the age of eighteen and over eighteen. The introduction of shared entities like media devices, data sources, social spaces and settings further highlight the many nuances of the interactions within this legal need that calls for immediate attention.

Reflected in the findings were the measures required for the realisation of the GDPR. Providing transparency about data practises was identified as a method for translating legislation into design. Currently, transparency is enabled through introduction of new websites that present information to users in simple and engaging ways, a shift in the format of the terms and conditions statement, general use of simple language when discussing data practises etc. Thus, realisation of transparency is a goal that is expected to be achieved through legible interaction strategies.

\section{Social}

The turn towards personal data in new media experiences highlights several challenges that places the call for better social accountability. Social accountability, while not regulated like in the legal scenario, is considered a requirement to help build user trust in personal data environments. It refers to being accountable as a societal entity, respecting and mitigating the social implications of using personal data that could lead to loss of user trust.

"And I am not sure how comfortable they would be in answering what is your ethnic background or your religious beliefs and things like that. I would like for us to deliver value for everyone but I'm not so sure that I would like to ask for that information. So, it's a rather tricky situation...[...]....to put it bluntly, it would be a bit creepy to ask for that kind of information.[P12]"

Fear of reputation damage, due to the organisation not being able to be fully accountable for its use of personal data was a challenge that triggered many actions, precautions and decisions. This fear is often associated with the fear of loss of user trust from the use of personal data and thus becomes a social challenge that constrains the complete realisation of the creative potential offered by data driven media.

Personal data can act as a tool that enables understanding the audiences or the society in many ways that help accurate delivery of appropriate content to users. It helps explore user wants and needs on an individual level and allows for uncovering cohort behaviours. However, knowing the balance of when to utilise information about user membership in a particular service-created cohort versus when to respect user individuality is a social challenge which requires further attention.

\section{Ethical}

Ethical considerations are responses to both legal and social accountability challenges. While not dictated, or regulated by any specific entity in the media space, ethical data practises have been recognised by service providers as a key necessity in building user trust in personal data ecosystems.

"I think that you can do it the right way and the wrong way. For me, I think that if you are asking something, if you are asking people for more information about who they are, or what they think about stuff, then you need to give something back, or you need to justify why you are asking for it.[P13]"

The possibility of using information about the audiences to constrain their consumption and bias and prime them to align with the service providers' wants and needs surfaced as an ethical challenge that could contribute considerably to the current crisis in trust[20]. Thus, the challenge of using the collected data in ethical ways and the effective communication of these interactions to build user trust demands further consideration.

While age appropriateness of broadcast is often legally regulated, with the expansion of media moving beyond just broadcast coupled with the previously discussed involvement of shared media consumption settings, the ethical need to regulate the appropriateness of the content served in these social scenarios is also highlighted.

Personal data is currently considered by some as an asset class[44] on the same lines as oil and gold, but in contrast to these fungible assets, personal data is highly contextual and raises the creative challenge of ethically utilising it to its fullest potential. The challenge then is to innovate as a service provider and provide services that are on par with the competition while not compromising on user trust through unethical behaviour. 


\section{Technological reasonings}

The response to some of these legal, social and ethical challenges are currently enabled through the use of technology. Thus, technology becomes a medium that embodies solutions that lead to more trustworthy data interactions.

Examples of such technological reasonings include data security where techniques such as anonymisation, data minimisation, encryption etc are adopted to avoid realising loss of user trust and reputation damage. Thus, investing in interactions that provide and communicate higher user data security are expected to lead to increased user trust.

Use of cohorts is a technological translation of the need to manage legal requirements, evaluate content consumption, deliver relevant content and identify and cater to underserved audiences. The challenge of recognising the difference between contexts that require cohort use and those where cohorts are dysfunctional requires further attention. Here, further research could make valuable contributions by developing interaction strategies that overcome erroneous stereotyping by exploring the socio technical effects of cohorts and identifying scenarios where they disempower versus empower users.

Transparency was highlighted as one of the legal requirements of the GDPR that is hoped to result in increased user trust. Terms and conditions statements are a classic example of transparency provision where despite active research in both industry[52] and academia[21,43], the adoption of alternative forms of privacy statements that empower the user, still remain a challenge. Thus, designing data interactions that lead to increased awareness and understanding of the underlying data practises are prioritised as a means for building user trust.

Using user personal data to understand audiences to help craft future content was discussed previously. The user is now a participant in the creative, editorial and commissioning processes, making future media experience design and dissemination procedures more user centric. Such initiatives that help address the concern of biases by involving actual user behavioural and consumption data in the crafting process are expected to increase the relevance of services and build user trust.

Providing meaningful feedback in the form of selfreflection summaries, quantified-self initiatives or visualisations that demonstrate the user's role in the digital economy have been identified as alternatives for trust creation. Helping the users feel more empowered through experiences that encourage user trust is expected to open potential avenues for improved value exchange.

\section{CONCLUSION AND FUTURE WORK}

The turn towards personal data in driving novel media experiences is resulting in changing priorities and challenges when delivering these experiences to audiences.
This interview study explores these current challenges and priorities of media service providers working on a diverse range of data driven media experiences within a large U.K. based media organisation.

The results of this study unpack a call from the service provider's perspective for improved mechanisms and interactions within the user-data-service ecosystem where trust and value are consistently upheld and balanced as priorities. This balance has been identified to be preserved through being legally compliant and going beyond just the mandatory to also ensure social accountability and ethical responsibility as an organisation. With media and broadcast ranging diversely in terms of content, services and audiences catered to, the manifestation of these priorities are diverse and highly context dependant. But here, technology is often seen as a solution for realising many of these diverse priorities successfully to ensure sustainability within the personal data ecosystem.

While this research uncovers the priorities and potential pathways that would ensure continued innovation in new media experiences driven by personal data, there is still the call to explore further practical alternatives that add value to the interactions between stakeholders within the ecosystem in a trusted manner. Previous research like Human Data Interaction[32] provides theoretical frameworks that support such interactions, through the recommendation of application of principles like data legibility, negotiability and agency. But, there is still the call for practical solutions and design recommendations that could be used in everyday contexts. To fully understand the technological and social viability of such alternatives we intend to further extend this research through the use of technological probes that involve personal data driven media experiences supported by alternative technological solutions like personal data management systems[1,23,33].

We are currently exploring an IoT data driven adaptive media experience contexualised in the living room of the home, which applies the legal, social and ethical priorities highlighted in this study. Here, the Databox, a personal networked device that allows users to regain agency of their online presence[16] through active control and management of their personal data, would be used to enable legal, ethical and socially accountable interactions to ensure trustworthy data transactions that users could actively engage with. We aim for such novel alternatives to probe audiences and solicit insights and practical possibilities for the future that provide value to both the user and the service provider in the personal data ecosystem, while preserving user trust.

\section{ACKNOWLEDGEMENTS}

This work was supported by the BBC R\&D and EPSRC grants EP/L015463/1 and EP/N028260/1.

\section{REFERENCES}

1. Yousef Amar, Hamed Haddadi, and Richard Mortier. 2016. Privacy-Aware Infrastructure for 
Managing Personal Data Personal Data Arbitering within the Databox Framework. Proceedings of the 2016 conference on ACM SIGCOMM 2016 Conference: 571-572.

2. Xavier Amatriain. 2013. Big \& personal: data and models behind netflix recommendations. Proceedings of the 2nd International Workshop on Big Data, Streams and Heterogeneous Source Mining Algorithms, Systems, Programming Models and Applications - BigMine '13, 1-6. http://doi.org/10.1145/2501221.2501222

3. Jonathan Bacon. 2016. Young viewers choosing VOD over pay TV. Marketing Week. Retrieved September $18, \quad 2017$ from https://www.marketingweek.com/2016/02/11/youn g-viewers-choosing-vod-over-pay-tv/

4. Douglas Benson and John A Hughes. 1983. The perspective of ethnomethodology. Longman Publishing Group.

5. Frank Bentley, Karolina Buchner, and Joseph "Jofish" Kaye. 2014. MyChannel: Exploring Citybased Multimedia News Presentations on the Living Room TV. Proceedings of the ACM International Conference on Interactive Experiences for TV and Online Video., ACM, 7178. http://doi.org/10.1145/2602299.2602302

6. Fernando Bermejo. 2009. Audience manufacture in historical perspective: from broadcasting to Google. New Media \& Society 1-2, 11: 133-154. http://doi.org/10.1177/1461444808099579

7. danah boyd and Kate Crawford. 2012. Critical Questions for Big Data. Information, Communication \& Society 15, 5: 662-679. http://doi.org/10.1080/1369118X.2012.678878

8. Virginia Braun and Victoria Clarke. 2006. Using thematic analysis in psychology. Qualitative research in psychology 3, 2: 77-101.

9. Eric Brynjolfsson, Lorin M Hitt, and Heekyung Hellen Kim. 2011. Strength in numbers: How does data-driven decisionmaking affect firm performance?

10. Matt Carlson. 2006. Tapping into TiVo: New Media \& Society 8, 1: 97-115. http://doi.org/10.1177/1461444806059877

11. Colin Cherry. 1977. The telephone system: creator of mobility and social change. The social impact of the telephone: 112-126.

12. Tony Churnside. 2013. Object-Based Broadcasting. Retrieved September 10, 2016 from http://www.bbc.co.uk/rd/blog/2013-05-objectbased-approach-to-broadcasting
13. Paula Falco, Christina Noonan, and Ge Cao. 2016. REFLEX: Face Micro-Expression Recognition System for TV Content Curation. Proceedings of the ACM International Conference on Interactive Experiences for TV and Online Video, ACM, 163169. http://doi.org/10.1145/2932206.2933564

14. Harold Garfinkel. 1996. Studies in Ethnomethodology. Polity Press, London, United Kingdom.

15. Article 7 GDPR. 2016. Regulation (EU) 2016/679 of the European Parliament and of the Council of 27 April 2016 on the protection of natural persons with regard to the processing of personal data and on the free movement of such data, and repealing Directive 95/46/EC (General Da. Official Journal of the European Union L119, 1-88.

16. John Haddadi, Hamed, Howard, Heidi, Crowcroft. 2015. Personal Data: Thinking Inside the Box. 8 .

17. Hilary Hutchinson, Wendy E. Mackay, Bo Westerlund, et al. 2003. Technology probes: inspiring design for and with families. Proceedings of the SIGCHI Conference on Human Factors in Computing Systems - CHI '03, 17-24.

18. Wijnand IJsselsteijn and Wijnand. 2017. Here's Looking At You, Kid. Proceedings of the 2017 ACM International Conference on Interactive Experiences for TV and Online Video - TVX '17, ACM, $1-1$. http://doi.org/10.1145/3077548.3077562

19. Iris Jennes. 2017. The Social Construction of Targeted Television Advertising: The Importance of "Social Arrangements" in the Development of Targeted Television Advertising in Flanders. Proceedings of the 2017 ACM International Conference on Interactive Experiences for $T V$ and Online Video, ACM, 41-50.

20. Carl Kalapesi. 2012. Rethinking Personal Data: Strengthening Trust.

21. Patrick Gage Kelley, Joanna Bresee, Lorrie Faith Cranor, and Robert W Reeder. 2009. A "nutrition label" for privacy. Proceedings of the 5th Symposium on Usable Privacy and Security SOUPS 09, 4. http://doi.org/10.1145/1572532.1572538

22. Pyungho Kim. 1999. A machine-like new medium - theoretical examination of interactive TV. Media, Culture \& Society 25, 2: 217-233.

23. Tom Kirkham, Sandra Winfield, Serge Ravet, and Sampo Kellomaki. 2013. The personal data store approach to personal data security. IEEE Security and Privacy 11, 5: 12-19. http://doi.org/10.1109/MSP.2012.137 
24. Christopher Krauss, Lars George, and Stefan Arbanowski. 2013. TV predictor: personalized program recommendations to be displayed on SmartTVs. Proceedings of the 2nd international workshop on big data, streams and heterogeneous source mining: Algorithms, systems, programming models and applications. ACM, 2013., 63-70.

25. Jaron Lanier. 2014. Who owns the future? Simon and Schuster.

26. Steve LaValle, Eric Lesser, Rebecca Shockley, Michael S Hopkins, and Nina Kruschwitz. 2011. Big data, analytics and the path from insights to value. MIT sloan management review 52, 2: 21.

27. Jonathan Lazar, Jinjuan Heidi Feng, and Harry Hochheiser. 2017. Research methods in humancomputer interaction. Morgan Kaufmann.

28. Karl Manheim. 1952. On the interpretation of Weltanschauung. From Karl Manheim.

29. M. Lynne Markus. 1987. Toward a "critical mass" theory of interactive media: Universal access, interdependence and diffusion. Communication Research 14, 5: 491-511. http://doi.org/10.1177/009365087014005003

30. Nan L Maxwell, Dana Rotz, and Christina Garcia. 2016. Data and Decision Making: Same Organization, Different Perceptions; Different Organizations, Different Perceptions. American Journal of Evaluation 37, 4: 463-485.

31. Aleecia M. McDonald and Lorrie Faith Cranor. 2008. The Cost of Reading Privacy Policies. $I / S$ - $A$ Journal of Law and Policy for the Information Society 4, 3: 1-22.

32. Richard Mortier, Hamed Haddadi, Tristan Henderson, Derek Mcauley, and Jon Crowcroft. 2014. Human-Data Interaction: The Human Face of the Data-Driven Society.

33. Min Mun, Shuai Hao, Nilesh Mishra, et al. 2010. Personal data vaults: a locus of control for personal data streams. Proceedings of the 6th International COnference on - Co-NEXT'10: 17.

34. Matthew Pike, Richard Ramchurn, Steve Benford, and Max L. Wilson. 2016. \# scanners: exploring the control of adaptive films using brain-computer interaction. Proceedings of the 2016 CHI Conference on Human Factors in Computing Systems, ACM, 5385-5396.

35. Roy Rada. 1995. Hypertext. In Interactive Media. Springer New York, New York, NY, 21-47. http://doi.org/10.1007/978-1-4612-4226-0_3

36. Dave Randall, Liz Marr, and Mark Rouncefield. 2001. Ethnography, ethnomethodology and interaction analysis. Ethnographic Studies 6, 1: 3144.

37. Joel R Reidenberg, Travis Breaux, Lorrie Faith Carnor, et al. 2014. Disagreeable Privacy Policies: Mismatches Between Meaning and Users' Understanding. Berkeley Technology Law Journal 30, 1: 39-88. http://doi.org/10.15779/Z384K33

38. Colin Robson. 1993. Real world research: A resource for social scientists and practitionersresearchers. West Sussex: John Wiley \& Sons.

39. John Rose, Olaf Rehse, and Björn Röber. 2012. The Value of our Digital Identity.

40. Harvey Sacks. 1984. Notes on methodology. Structures of social action: Studies in conversation: 21-7.

41. Harvey Sacks. 1992. Lectures on conversation. 2 vols. Edited by Gail Jefferson with introductions by Emanuel A. Schegloff.

42. Neelima Sailaja, Andy Crabtree, and Phil Stenton. 2017. Challenges of using personal data to drive personalised electronic programme guides. Proceedings of the 2017 CHI Conference on Human Factors in Computing Systems, ACM, 5226-5231.

http://doi.org/http://dx.doi.org/10.1145/3025453.30 25986

43. Florian Schaub, Rebecca Balebako, Adam L Durity, and Lorrie Faith Cranor. 2015. A Design Space for Effective Privacy Notices. Eleventh Symposium On Usable Privacy and Security (SOUPS 2015), 1-17.

44. Klaus Schwab, Alan Marcus, J. O. Oyola, William Hoffman, and M. Luzi. 2011. Personal Data: The Emergence of a New Asset Class.

45. Paul M Schwartz and Daniel Solove. 2009. Notice \& Choice.

46. Ryan Singel. 2009. Netflix Spilled Your Brokeback Mountain Secret, Lawsuit Claims. Wired. Retrieved September 18, 2017 from https://www.wired.com/2009/12/netflix-privacylawsuit/

47. Ryan Singel. 2010. NETFLIX Cancels Recommendation Contest after Privacy Lawsuit. Retrieved March 29, 2018 from https:/www.wired.com/2010/03/netflix-cancelscontest/

48. Jonathan Steuer. 1992. Defining Virtual Reality: Dimensions Determining Telepresence. Journal of Communication 42, 4: 73-93. http://doi.org/10.1111/j.1460-2466.1992.tb00812.x

49. European Data Protection Supervisor. 1995. Data 
Minimization Article 6.1(b) and (c) of Directive 95/46/EC. Retrieved from https://edps.europa.eu/node/3080

50. European Data Protection Supervisor. 2017. Data Minimization Article 4.1(b) and (c) of Regulation EC (No) 45/2001. Retrieved from https://edps.europa.eu/taxonomy/term/133

51. Amy B Wang. 2017. Trump renews attack on "Fake News CNN" after retraction. Retrieved March 29, 2018

from

https://www.washingtonpost.com/news/the-

fix/wp/2017/06/27/trump-renews-attack-on-fakenews-cnn-after-

retraction/?utm_term $=.6 \mathrm{a} 2087289 \mathrm{e} 12$

52. Zynga. 2011. PrivacyVille. Retrieved September 18, 2017 from https://www.zynga.com/privacy/privacyville 